(hemischen Reaktionen widerstandsfähiger ist als das reine Bromsilber

Mit den im vorstehenden angedeuteten Momenten glaube ich die Rolle der Gelatine in den photographischen Schichten insofern einigermaBen erschöpfend behandelt $z u$ haben, als ich diejenigen Punkte einer Diskussion unterwarf, die ganz spezielle, auch für die Praxis der Emulsionsbereitung wichtige photographische Probleme darstellen. Daß auch die allgemeineren Diffusionsvorgänge in Gallerten zu aller-

Zeitschr. 1, 353, 1906). Luppo-Cramer, Kolloidchem. u. Phot. (Dresden 1908), 121. hand Komplikationen Anlats geben, die $\%$. B. einen wesentlich anders gearteten Entwicklungsvorgang bei Kollodiumplatten als bei Gelatineplatten bedingen, ist selbstverständlich und kann gelegentlich in anderem Zusammenhange besprochen werden. Schon das geschilderte Material wird aber dem der photographischen Spezialwissenschaft fernerstehenden Leser gezeigt haben, wie viel auch in dieser Richtung zu tun noch übrig bleibt, um die wissenschaftliche Beherrschung eines technisch auf ziemlich hoher Stufe stehenden Fabrikationszweiges zu ermöglichen und die diffizile Emulsionsmacherei aus ihrem "empirischen Elend" zu erlösen.

\title{
Kolloidchemie der Brauereiwissenschaft im Jahre 1911.
}

\section{Von Richard Emslander (Stuttgart). (Eingegangen am 4. Pebruar 1912)}

Ein flüchtiger Blick in die Literatur des verflossenen Jahres zeigt, da $B$ alle Bestrebungen, alle Forschungswege der Brauereiwissenschaft zusammenlaufen in dem einen, großen Ziel, das sich "Azidităt" nennt. Kaum eine andere Frage ist in der Tat besser geeignet, dem Kolloidforscher reichliche Ernte zu verheifen. In klarer Erkenntnis der hochwichtigen, aktuellen Angelegenheit hat W. Winldisch an den SchluB seines Vortrages - gehalten auf der Oktobertagung der Versuchs- und Lehranstalt für Brauerei in Berlin -- die Forderung gestellt:

"Es sind an den wissenschaftlichen Brauereiinstituten die Mittel bereitzustellen zur Beschaffung der Einrichtungen und Verfügbarmachung der Arbeitskräfte für physikalischchemische Forschungen, die die Aufgabe haben, das für die Brauerei so ungemein wichtige Gebiet der Kolloidchemie zu bearbeiten, also die Fragen zu behandeln der Enzymologie, der wirklichen und Scheinlosung, Ausflockung, Bruchbildung, Klärung, Entbitterung, Vollmundigkeit, Schaumhaltigkeit, Haltbarkeit, soweit sie durch chemisch-physikalische Einflüsse beherrscht ist, Kăltebeständigkeit, Pasteurisierfähigkeit -.- alles ungemein wichtige Fragen, über die wir noch im dunkeln tappen und die der wissenschaftlichen Klärung durchaus bedürfen, alles Fragen, die mit der Azidität und damit aufs engste mit der Beschaffenheit des Brauwassers zusammenhängen. “

F. M. Fernbachy. Dle Bedeutung der Phosphorsäure und ihrer Verbindungen in der Brauerei.
Bei der Gärung der Bierwürze spielt auBer den vergärbaren Zuckern noch eine Reihe wichtiger anderer Faktoren eine große Rolle, so besonders die Mineralsubstanzen, die aus dem Rohmaterial in die Würze kommen. Die Asche der Gerste, die im Mittel $2,6 \%$ des Korngewichtes beträgt, setzt sich in der Hauptsache aus Phosphorsäure zusammen, die als Kaliumsalz ungefähr $55 \%$ der Asche ausmacht. In der Pflanze ist die Phosphorsäure an organische Substanzen gebunden als Phytin; dieses Phytin wird bei der Keimung der Gerste durch die Phytase abgebaut, wobei sich anorganische Phosphorsäure unter Umsetzung au phosphorsauren Salzen bildet.

Die Phosphate wirken ein auf den Maischprozeb und auf die Gärung.

Kjeldahl hat als erster die Beobachtung gemacht, daf, wenn man eine Săure in steigenden Mengen zu einer Mischung von Stärke und Malzauszug hinzufügt, die GröBe der in der gleichen Zeit gebildeten Maltosemenge in demselben Verhältnis wăchst, wie man die Săuregabe vergröBert, bis ein bestimmter Säuregrad erreicht ist, von dem ab dann wieder eine $\mathrm{Ab}$ nahme der fördernden Wirkung der Säure eintritt. Die Phosphate spielen zweifellos bei der Tätigkeit der Amylase des Malzes eine ganz hervorragende Rolle, indem sie als neutrale oder sekundäre Salze deren Wirksamkeit beeinträchtigen, während sie als saure oder primăre

1) Vortrag, Annales de la Brasserie et de la Destillerle 14, 217 . 221 (1911). Uebersetzt von $H$. Schnegg Zeltschr. f. d. ges. Brauw. 1911, 359 u. है. 
Phosphate eine günstige Wirkung ausühen. Ein Malz wird daher heim Maischprozeb um so mehr Maltose liefern, je größer sein Gehait an primären Phosphaten, $d$.h. je höher sein Săuregrad ist.

Während der Keimung der Gerste verwandeln die organischen Säuren, wenn sie mit löslichen sekundären Phosphaten zusammentreffen, diese in primäre oder saure Phosphate. Dadurch entstehen im wesentlichen jene Verbindungen, welchen die Würze ihre Säure verdankt. Diese sauren Phosphate neutralisieren in zweiter Linic auch noch die alkalisck reagierenden Salze, besonders die Bikarbonate, welche durch das Wasser in die Würze gelangen und die normale Verzuckerung beeintrăchtigen würden. Die Phosphate sind demnach der Regulator der enzymatischen Vorgänge beim Maischprozef.

Bei der Gärung der Würze ist ihre Aufgabe nicht minder bedeutend. Vor allem sind sie ein wichtiger Nährstoff der Hefe, deren Asche ja allein zu $85.7 \%$ aus Phosphorsäure $\left(\mathrm{P}_{2} \mathrm{O}_{5}\right)$ besteht. Die sauren Phosphate stellen andererseits, da auf ihnen zum Teil die Säure des Bieres beruht, ein Element dar, das für die Haltbarkeit des Bieres von gröflerer Bedeutung ist als die neutralen Phosphate. Es ist darum vielmehr dic Art der Phosphate ausschlaggebend und nicht so sehr die Quantitat ohne Berücksichtigung, ob primärer oder sekundärer Natur.

Der HefepreBsaft enthallt zwei Arten von Stoffen, die in Gemeinschaft aktiv sind, d. h. in Zuckerlösung Gärung erzeugen; werden diese beiden Teilhaber aber durch Ultrafiltration voneinander geschieden, so vermag keiner allein eine Wirkung in dem angegebenen Sinne auszuüben, sie beide sind getrennt inaktiv. Der eine dieser beiden Gesellen, das Ko-Enzym nach Harden und Young, ist hitzebeständig und vermag, wenn er mit seinem Teilhaber zusammengebracht wird, diesen wieder zu neuer Tatigkeit anzuspornen, zu reaktivieren. Dieses Ko-Enzym besteht nun aus nichts anderem als aus Verbindungen der Phosphorsäure mit organischen Korpern, es ist nach $\mathrm{Buchner}$ ein Ester, der durch Lipase verseifbar ist. Harden konnte einwandfrei nachweisen, dat ein Zusatz von Dinatriumphosphat zu Hefeprebsaft dessen Gärtätigkeit proportional der zugesetzten Menge erhöht. Unter diesen Umständen ist ein Waschen der Hefe mit Phosphorsüure von eminenter Bedeutung, sie desinfiziert also nicht allein, sondern hauptsăchlich reaktiviert sie die Zymase.
R. Forster ${ }^{2}$ ). Ueber Bitterstoffe, spezjell Hopfenbitterstoffe und ihre medizinische Verwendung.

Verfasser bespricht zunächst die Wirkung der wichtigsten Bitterstoffe auf den menschlichen und tierischen Organismus. Salizin und Phlorrhizin wirken weder auf die Stärkeverdaunng im Munde noch auf die Eiweibverdauung im Magen günstig ein. Der Magensaft allein löste mehr EiweiB, als bei Zusatz von Chinin, Berberin, Absinthin und Phlorrhizin gelöst wurde. Nach J. Traube soll die Spannung im arteriellen Bltstkreis eine Steigerung erfahren, die sich nach mäßBigen Gaben von Cetrarsăure, Kolombin durch geringes Absinken und allmähliches $\mathrm{An}$ steigen des arteriellen Blutdruckes bemerkbar mache. Quassiin soll vernichtend auf niedere Organismen, insbesondere auf Gärungserreger, wirken. Nach einer Zusammenstellung von Bernat $z$ ki über Gesundheitsstörungen kommt Verfasser auf die Bitterstoffe des Hopfens zu sprechen. Die kristallisierbare Hopfenbittersăure $\mathrm{C}_{25} \mathrm{H}_{36} \mathrm{O}_{4}$ ist, dem Blute direkt einverleibt, nach Tappeiner sehr gifrig; sie beschleunigt zunächst die Atmung und lähmt sie dann. Durch den Mund zugeführt ist der Stoff dagegen wirkungslos, weil er sehr rasch $\mathrm{zu}$ amorphem Bitterstoff zersetzt wird. Im Biere ist nur letzterer zu finden. Eine wesentliche Eigenschaft des Hopfenbitters ist seine beruhigende, nach einigen sogar einschläfernde Wirkung. Die schläfrigmachende Wirkung des Bieres soll auf dessen Gehalt an Hopfenbestandteilen zurückzuführen sein. Das erste Hopfenpräparat wurde 1813 von dem Pariser Apotheker Planchet unter dem Namen Lupulin in den Handel gebracht. Es wurde gegen Erregungszustănde der Sexualorgane verwendet. Auch bei Blasenleiden und Gonorrhöe wird Lupulin als Beruhigungsmittel empfohlen. Als Magenmittel leistet das Hopfenbitter hauptsächlich gegen mangelnden Appetit und bei Magenkatarrh nach Ablauf der akuten Erscheinungen gute Dienste. Ferner findet Hopfen noch Anwendung als Zugabe zur Digitalistinktur bei Herzleiden sowie bel Tuberkulose.

Adrian J. Brown und G. Bernerd Ward ${ }^{3}$ ). Ueber die Bewertung der antiseptischen Eigenschaften des Hopfens.

Die antiseptische Wirkung des Hopfens schwankt innerhalb sehr weiter Grenzen. Ver-

ף) Wochenschr. f. Brauerel 1911, 163.

3) Ref. Zeitschr. f. d. ges. Brauw. 1911, 314. O. A. Journ. Inst. Brew. 1911. 
fasser arbeiteten eine Methode aus, am den relativen Wert eines Hopfens in bezug auf seine antiseptischen Eigenschaften zu beurteilen. Sie fanden in einem saueren Bier ein dem Saccharobazillus pastorianus Van Laers ähnliche Bakterium, das sich durch ihre auBerordentliche Fuhigkeit, sich in einem geeigneten flüssigen Medium zu vermehren und ihre ungemeine Empfindlichkeit gegen den toxischen Einflub des Hopfenextraktes auszeichnete. Diese Eigenschaften dienten den Verfassern zur Messung der antiseptischen Eigenschaften des Hopfens. Die toxische oder antiseptische Wirkung eines Hopfens wird ausgedrückt auf einer Grundlage von 100, welche die toxische Kraft bezeichnet, so daB $0,25 \mathrm{ccm}$ des Normalhopfenextraktes das Wachstum des Organismus verhindert. Die folgenden sind typische Beispiele der mit verschledenen Hopfen des Jahres 1909 erhaltenen Resultate :

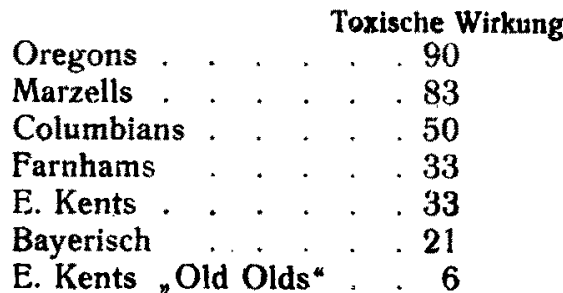

Die Weichharze besitzen eine $50 \mathrm{mal}$ grobere toxische Wirkung als die Hartharze, wahhrend die Infusionen aus dem ausgezogenen Hopfen überhaupt keine toxische Wirkung mehr besitzen. Es wird die Vermutung ausgesprochen, daB die toxische Wirkung der Hartharze von der Gegenwart einer Spur des aus der Extraktion mit Petroläther abriggebliebenen Weichharzes herrühren mag. Es wurde ferner gefunden, daA, wenn der Organismus längere Zeit dem Einflusse eines starken Hopfenextraktes ausgesetzt wurde, das Leben des Organismus nicht gestort wurde, sondern daB derselhe bei nachfolgender Einimpfung in ungehopfte Kulturmedia sich normal entwickelte. Dies zeigt, dab der Hopfenextrakt nur das Wachstum des Organismus verhindert, ohne tödlich auf denselben einzuwirken.

F. Schönfeld und W. Hirt ${ }^{4}$ ). Die Zinnempfindlichkeit bei hellen Bieren. (Metalleiweib. trübung.)

Die Widerstandsfähigkeit gegen Zinn ist bei den verschiedenen Bieren verschieden. Die Biere ein und derselben Brauerei weisen meistens ein ziemllch gleiches Verhalten auf. Die Ausscheidungen von Trübungen sind stärker bei

4) Wochenschr. f. Brauerei 1910, 653 . hohem Kohlensäuregehát der Biere als bei Mangel oder Fehlen. der Kohlensäure. (Dies dürfte daher rübren, daB die Trübung verursachenden Suspensionskolloide beim Entweichen der Kohlensäure ihren Stabilisator, nämlich die Kohlensäure, verlieren, zusammenvallen und koagulieren. Referent.) Zwischen Stärke der Trübung und Säuregehalt besteht insofern eine Beziehung, als die säureärmsten Biere die widerstandsfähigsten, die säurereichsten dagegen die empfindlichsten sind.

Durch Abstumpfen der Säure mittels Alkalien wird die Ausscheidung von Trübungen mehr oder minder stark herabgesetzt und manches Bier, das zu Trübungen neigt, vollständig zinnfest gemacht, so daB es blank bleibt. Die săurereichsten Würzen lieferten die säurereichsten und auch empfindlichsten Biere und utngekehrt die säureärmsten die widerstandsfähigsten Biere. Stumpft man den Săuregehalt der Würze durch kohlensauren Kalk ab, oder kocht man die Würze nach Entfernung des Trubes, so sind die aus den so behandelten Würzen bereiteten Biere widerstandsfähiger als die aus den Originalwürzen bereiteten. Die Zusammensetzung des Brauwassers ist von grobem Einflub auf die Empfindlichkeit des Bieres; karbonatreiche Wasser geben wenig empfindliche Biere, sulfatreiche stark empfindliche. (Es fragt sich nur, ob diese zinnfesten Biere auch vollmundig und nicht leer sind; denn es ist sehr wahrscheinlich, daf durch Neutralisation sämtliche (koagulierbare) Geschmackstoffe entfernt werden, so dab eine grobe Unempfindlichkeit hervorgerufen werden kann. Referent.)

A. Reichard ${ }^{5}$ ). Studien über Bierkolloide,

Die sogenannte panoptische Triazidlösung nach A.Pa p pe n beim, eine Mischung von blauem basischen Farbstoff, rotem Säurefuchsin, sowie Săureorange, färbt Gerstenschnitte teils rot, teils blau und grün. Rot wird das EndospermeiweiB, blau und grün die Randzone des Gerstenkornes. Ersteres bekundet dadurch seinen basischen, letztere ihren sauren Charakter. Verfasser studierte an Gerstenschnitten die Färbungen der einzelnen Gewebeteile năher, um dadurch Ausscheidungen aus ungehopften Würzen, Treberteilchen, Treberprebisaft bezüglich ihrer näheren Herkunft feststellen zu können. Dabei fand er, daß sich die Aleuronzellenschicht beim Mälzungs- und BrauprozeB nicht ganz so passiv verhalten dürfte, wie ziemlich allgemein angenommen wird. Es findet 'vielmehr eine

5) Zeitschr. f. d. ges. Brauw. 1911, Nr. 21 u. 22. 
Fettdispersion und Uebergang der Emulsion in die Würze statt, insbesondere dann, wenn die Treber unter Druck erhitzt werden; auch könten unter Umständen die Bedingungen zur Seifenbildung gegeben sein.

Die beobachtete starke Blaufărbung des Gerbstoffs der Samenhaut veranlaften den Verfasser, mit Triazid auch Färbungsversuche in Flüssigkeiten zu machen, in denen eben Gerbstoff gelöst war. In der Tat ergab sich auch hier eine Fàrbendifferenzierung, indem die ursprünglich violette Färbung des Triazidgemisches (durch frei gewordenes Fuchsin) rot wurde, während der blaue Farbstoff von dem emulgierten Gerbstoffteilchen adsorbiert wurde, wodurch die Flüssigkeit einen blauen Schiller annahm. Die Stärke dieses Blauschillers lief parallel mit dem Dispersitătsgrad des Gerbstoffes der Lösung, $d$. h. je niederer der Dispersitätsgrad desto stärker die Blaufärbung. Auch die üblichen Gerbstoffreaktionen mit Eisensaizen und mit Goldchloridchlornatrium $(0,15$ prozentig $)$ veränderten je nach dem Dispersitätsgrad dt: Gerbstoffs ihre Nuancierung, wie das leicht zu beobachten ist bei starker Verdünnung der Reagenzıen. Der Dispersitätsgrad wird erhöht durch Essigsäure und auch durch längeres Kochen der wässerigen Gerbstofflösung, im Gegensatz zu kalter Lösung und noch mehr zu alkoholischer Losung; aus Alkohol lieB sich der Gerbstoff durch Aether oder Chloroform koagulieren; insbesondere ließen sich durch das Tyndallphänomen gröbere Massenteilchen (Gerbstoffkoagula) erkennen. Typisch ist die Reaktion mit Eisenchlorid insofern, als bei geringem Dispersitätsgrad des Gerbstoffs tiefblaue Farbtöne auftreten, während bei höherem Dispersitătsgrad desselben eine blasse, mehr violette oder grünliche Tönung zu sehen ist. (Die hohe brautechnische Bedeutung des Dispersitätsgrades von Hopfengerbstoff wird durch den Befund des Verfassers, daß der kaltgelöste [= höher kolloide] Gerbstoff gröbere Eiweißkoagula gibt und so die Würze besser klärt als der Gerbstoff mit höherem Dispersitătsgrad oder feinerer Verteilung, markant bewiesen. D. Ref.;

Aehnlich wie Gerostoff färben sich auch die in wässerigen Hopfenauszügen emulgierten Hopfenharztröpfchen mit der Triazidlösung blau unter Dissoziierung der Farbmischung in blaue und rote Komponenten. Alkoholische Hopfenharzauszüge dissoziieren trotz höheren Harzgehaltes das Triazid weit weniger sturk (ein ge- ringer Blauschiller!). Die Ursache davon ist die, dak der Emulsionscharakter der wässe. rige $n$ Lösung ein ausgesprochener ist, während der Alkoholauszug den Charakter einer reinen Lösung besitzt; mit Wasser zusammengebracht entsteht jedoch auch hier eine Emulsion, ersichtlich an dem Auftreten blauer Opaleszenz; doch zeigt das Mikroskop, daf hier die Harztropfen grob suspendiert sind, während die Dispersion in der wässerigen Lösung (Emulsion) eine weitaus feinere ist. Daraus erklärt sich die bessere Schaumbildung wässeriger Hopfenharzextraktionen gegenüber alkoholischen; ähnliches hat auch F. Emslander beobachtet.

Der Gerbstoff des Hopfens ist weniger kolloid als der Gerbstoff der Gerste, denn er lieb sich nicht durch Aetherausschüttlung koagulieren und farbte sich mit Eisensalzen nicht dunkelblau, sondern grün. Von den beiden Repräsentanten des Hopfengerbstoffs, dem Phlobaphen und dem eigentlichen Gerbstoff, färbte sich ersterer stärker grün als letzterer, und diese Tatsache entspricht hinwiederum deren Verhalten zu EiweiB, indem der starkere Eisen-Reaktion gebende Gerbstoff das EiweiB auch besser ausflockt. Der Dispersitätsgrad ist also bei Beurteilung der Gerbstoffe hinsichtlich ihres Flockungsvermögens für Eiweib von ganz wesentlicher Bedeutung. Der Malzgerbstoff wird aus den Zellgeweben nur schwer extrahier;, durch Hitze allerdings leichter als in der Kälte; er dürfte während des Sudprozesses seine Fällungswirkung auf EiweiB ausüben. Seine chemische Aktivität ist geringer als die des Hopfengerbstoffs, d. h. er braucht längere Zeit zum Eingehen der EiweiBverbindung. LäBt man ihm durch längeres Kochen der ungehopften Würze Zeit zur Einwirkung auf deren Eiweibstoffe und kocht erst dann mit Hopfen, dann scheidet sich mehr GesamteiweiB ab, als wenn man sofort mit Hopfen kocht. Das gleiche that auch Seyffert ${ }^{6}$ ) beobachtet, und J. C. Lintnert) fand, das so behandelte dunkle Würzen widerstandsfähiger gegen Kälte wurden; bei bellen war dies weniger der Fall, was A. Reichard damit erklärt, daß helle Malze infolge ihrer geringen Zerreibbarkeit und daher gröberen Schrotbestandteile ihren Gerbstoff schwieriger hergeben.

Der Alkohol führt den Gerbstoff in größere Massenteilchen über, erhoht aber trotzdem nicht

6) Wochenscht. f. Brauerel 1904, Nr. 32.

7) Zeltschr. f. d. ges. Brauw. 1908, 525 . 
seine Komplex fallungswirkung gegenüher Eiweib: er ist dieser im Gegenteil hinderlich, da sich die entstehende Verbiädung in Alkohol löst.

G. Thevenot. Die Herstellung kältebeständiger Biere in den Vereinigten Staaten ${ }^{8}$ ).

Der Verfasser weist darauf hin, dab es in den Vereinigten Staaten Sitte ist, Flaschenbier in auberordentlich kaltem Zustande, fast bei Gefriertemperatur, auszuschenken und dab unter diesen extremen Bedingungen die meisten Biere, die einige Zeit in der Flasche gewesen sind, Schleier oder Trübung entwickeln. Je niedriger die Temperatur und je länger die Kühldauer, um so ausgesprochener wird in der Regel die Trübung sein. Schon seit Jahren sind daher Methoden im Gebrauch, die darauf hinzielen, ein Bier herzustellen, das die gewünschte Eigenschaft besitzt, beim Ahkühlen oder "Chilling“" glänzend zu bleiben. Verfasser unterscheidet zwei Trübungen in pasteurisiertem Bier, welche auf Temperaturwechsel zurückzuführen sind, die Trübung oder der Bodensatz, der bei höherer Temperatur, d. h. bei Zimmertemperatur, auftritt, und der mehr oder weniger ausgeprägte Schleier beim Abkühlen des Bieres durch Auftreten der sogenannten EiweiBtrübung infolge der allmahlichen Koagulation on FiweiBstoffen. Diejenigen Eiweikkörper, die bei höheren Temperaturen ausfallen, lösen sich nicht mehr imbier auf, einerlei, welchen Temperaturen man sie aussetzt. Nimmt man jedoch ein Bier, das durch starkes Abkühlen schleirig geworden ist, und erwärmt es nur schwach, nur um wenige Grade über den Gefrierpunkt, so varschwindet die Suspension, sie löst sich wieder auf und das Bier, erscheint wieder vollikommen klar, wenigstens in den meisten Fällen. Das Koagulieren der Eiweißkörper infolge zu hohen Pasteurisierens muB als eine primäre chemische Reaktion betrachtet werden. Andererseits dürfte die Koagulation von Eiweiß beim Abkühlen nur als eine mechanische Umwandlung in den unlöslichen Zustand anzusehen sein. (Das Bier ist bei sehr niederer Temperatur übersättigt an gelöstem EiweiB und gibt infolge von Zusammen. ballen des emulsoiden EiweiBes Trübung, welch letztere beim Erwärmen wieder verschwindet, da das Emulsionskolloid in den früheren Zustand zurïckkehrt. D. Ref.) Bezüglich der Theorien, die zur Erklärung der Kälteempfindlichkeit von Bieren aufgestellt worden sind, und der Methoden

8) Allgem. Brauer- u. Hopfen-Zeitung 1911, 540 Ref. Zeitschr. f. d. ges. Brauw. 1911, 236; aus O. A. American Brewer 1911, Nr. 2. der Herstellung kältcheständiget Blet fuht der Verfasser folgendes an: Man kann versuchen, ein Bier herzustellen, das fast kein odet nur sehr wenig unerwünschtes Eimeil ththall, $d . h$. solches Eiweib, das spater Triihung hervortufen könnte, oder man versucht, solches Eiveil3 unter allen Bedingungen in Lösung ou halten. Beide Methoden sind in Gebrauch, hauptsachlich die erstgenannte. Eine grolse Rolle spielt hierbei die Azidität des Materjals und hesondets die Alkalinität des Maischwassers, wie Versuche von Ed. Monfang, P. Petil und F. Emslander zeigten. Ferner soll die Kohlensăure und der atmosphärische Sauerstoff von grokem EinfluB auf die Haltbarkeit des Bieres sein. Da die Herstellung eines käitebeständigen Hieres durch die Entfernung der unerwünschten Eiweiß3körper nicht als eine ideale Behandlungsweise des Bieres angesehen werden kann, dürfte es vorteilhafter erscheinen, diese EiweiBkörper nach Möglichkeit in Lösung $z u$ erhalten und dem Biere eine derartige Zusammensetzung zu geben, dab sie nicht so leicht ihre kolloide Natur verlieren. Versuche in dieser Richtung führten dazu, dem Biere vor dem Pasteurisieren verschiedene Zusätze zu geben, wie verdünnte Milchsäure, Gerbsäure, Tragantgummi, Hopfenextrakte, Mischungen von Lupulin und Rohrzucker, Hopfenharzlösungen. Verfasser erzielte mit einer Anzahl der verwendeten Präparate sehr zufriedenstellende Resultate, doch sind die Versuche hierüber noch nicht abgeschlossen. (Neuerdings wird darch Pepsinzusatz das Uebel am gründlichsten behoben. D. Ref.)

Karl Maurer"). (leber den Einflub von sulfatreichen Brauwăssern auf die Qualitāt und Farbe des Bieres.

Verfasser gibt die Analyse zweier verschiedener Wasser, von denen das eine einen ungefähr achtmal gröBeren Sulfatgehalt aufweist als das andere. Dieses sulfatresche Wasser bewirkt eine ganz bedeutende Aufhellung der Bierfarbe; die Farbentiefe ist 0,6, während das sulfatarme Wasser dem Biere eine Farbentiefe von 1,2 verleiht. Als Erklärung dieser Tatsacht bringt der Verfasser die Aussalztheorie organischer Farbstoffe durch organische Salze. .Ditit Erklärungsweise hat viel Bestechendes für sich. doch gilt dieselbe streng nur für konzentrierte Salzlösungen, wie jeder Organiker zugeben mulb. $\mathrm{Da}$ aber zur Bierbereitung nur hochverdünite Salzlösungen angewandt werden, so kann für eire Farbbeeinflussung auch nur eine Frklärung.

9) Zeitschr. f. d. ges. Brauw. 1911, 589. 
die über verdünnte Salzlösungen etwas aussagt, Geltung haben. Auf der Suche nach einer derartigen Theorie ist es ganz besonders die Theorie der Kolloide, die man findet. Nach einem von $\mathrm{Karl} \mathrm{Maurer}$ angestellten Versuche ist die Aufhellungswirkung des Bieres bei Zusatz von Sulfat eine weit größere als die eines Chlorides. (Ein Blick in das schöne Werk Wo. Ostwald's, ,GrundriB der Kolloidchemie', II. Auflage 1911, zeigt, daß die innere Reibung der Gelatine (Emulsionskolloide) durch $\mathrm{SO}_{4}$ " bedeutend mehr erhöht wird als durch Chloride und durch Nitrate $\left(\mathrm{SO}_{4}{ }^{\prime \prime}>\mathrm{Cl}^{\prime}>\mathrm{NO}_{3}{ }^{\prime}\right)$. Wo. Ostwald gelangt auf Seite 217 dieses Werkes zu folgendem Satz: ,Im allgemeinen wird man theoretisch zu der Annahme neigen, $\mathrm{daB}$ die Viskosităt eines Dispersoids um so grö Ber sein wird, je größer die Berührungsfläche zwischen disperser Phase und Dispersionsmittel, m. a. W., je größer der Dispersitäts grad ist. Andererseits kann gemäß Beobachtungen die Viskosität auch zunehmen mit abnehmendem Dispersitătsgrad. $\mathrm{Ob}$ in diesem Spezialfalle die allgemeine Annahme gerechtfertigt ist, lafbt sich noch nicht mit absoluter Sicherheit sagen, da ja die Parbe des Bieres auch von Kristalloiden abhangig ist, doch hat sie die gröbere Wahrscheinlichkeit für sich." Säuren erhöhen bekanntlich den Dispersittatsgrad, Alkalien verringern denselben und demgemäßB bestatigt sich hier das Wo. Ostwald'sche Gesetz (Kolloidchem. Beih. 2, 12, 1911), daB sich das Absorptionsmaximum des Lichtes bei steigendem Dispersitätsgrad nach den kïrzeren Wellenlāngen verschiebt und umgekehrt. Was die Losung befördert, hellt auf, was dieselbe verringert, verdunkelt Wïrze und Bier oder erzeugt wenigstens den gefürchteten Rot- oder Grünschleier, ohne damit behaupten zu wollen, dab letztere Wirkung nicht auch noch andere Ursachen haben könnte. (D. Ref.)

E. Jalow et $\mathrm{z}^{10}$ ). Entkarbonisieren von Brauwasser.

Das Verfahren der „Entkarbonisierung" besteht nach dem Patente des Verfassers darin, karbonathaltiges Wasser mittels besonders intensiv wirkender Rührvorrichtungen gründlichst durchzuwirbeln, auf hohe Temperaturen zu überbitzen und unter Ueberdruck zu setzen. Die Karbonate des Kalkes und der Magnesia werden fast vollständig in kristalliner Form gefallt, auch Eisensalze werden quantitativ entfernt. Die übrigen etwa vorhandenen Salze bleiben unverandert.

10) Zeltschr. $f$ d ges Brauw. 1911, 5C9.
Die Karbontate färben das Bier stark zu. Dic: erklärt sich aus der Tatsache, dab der wasserige Hopfenextrakt der karbonatreichen Wasser rot ist, während der des entkarbonisierten Wassers grïn ist.

Der Schaum hält sich beim Karbonatwasser viel lânger als beim entkarhonisierten Wasser. Die gute Schaumbildung, eine Kolloidwirkung, konmt aber bei entkarbonisiertem Wasser, das wegen der helleren Farbe für helles Bier Verwendung findet, wieder zur Geltung, falls man verhältnismäBig mehr Hopfen zugibt, was in der Tat bei hellen Bieten zu geschehen pflegt. Die Dosierung der Hopfengabe richtet sich also ganz exakt nach dem Karbonatgehalte des zu verwendenden Wassers.

Oldřich Mišk ovsk(ł"). Ueber den Einflub des Brauwassers auf die Eigenschaften des Bieres.

Der Autor untersucht den EinfluB von schwefelsaurem, kohlensaurem, salpetersaurem und Chlorkalzium, ferner schwefelsaurem, kohlensaurem und Chlormagnesium sowie Chlornatrium in einer Konzentration von 0,1 g-Aequjvalent pro 1 Liter Wasser; er vergleicht die Wirkung dieser Salze mit jener des destillierten Wassers und bespricht bei gehopfter und nicht gehopfter Würze :

die Verzuckerungsdauer,

die Farbe der Wärze,

die Alkalität die Azidität der Reaktion,

den Extraktgehalt und

den Gesamt-Stickstoff in $50 \mathrm{ccm}$ der.Würze.

Die Verzuckerung trat bei Gipswasser, Chlormagnesium und Kalziumnitrat am schnellsten ein; bei Karbonat und Bikarbonat war die Verzuckerung steigend mit dem Gehalt entsprechend langsam, ein Befund, der ganz sicher ist, weil bereits von einer ansehnlichen Reihe von Forschern dargetan.

Die Farbe wird dunkler durch Karbonate sowohl in der Würze wie beim Hopfenkochen, jedoch heller durch Sulfate, besonders durch Gips; Gips paralysiert den Einflue von Karbouten.

Die Reaktion ist ausschlaggebend, steht doch die Tatsache fest, dab bei Neutralisation des Gesamt-Alkalis im Bier die Entwicklung der Sarzine bedeutend sinkt, ja vollstăndig aufhört. Die Azidität macht das Bier immun gegen Bakterien und widerstandsfähig gegenüber tieferen Temperaturen. Der Verfasser leitet aus

11) Zeilschr. f. d. ges. Brauw. 1911, Nr. 5, 6, 7, 8, 9. 
seiner Erfahrung den Schlub ab, daf die elektrometrische Titration der Würze an Schärfe viel zu wünschen übrig lasse. (, Auch ich glaube nach einigen Kontrollanalysen feststellen zu können, dab allerdings die Schärfe leidet, jedoch scheint mir gerade dieser Umstand davon herzurühren, daB in der Würze nicht e ine Säure oder e in sauer reagierendes Salz titriert wird, sondern deren mehrere; ich fand nämlich, dab bei Berücksichtigung der beiden hier sich ergebenden Kurvenäste eine vollständige Uebereinstimmung dieser Titrationsmethode mit jener nach E. Prior, wo bekanntlich Phenolphthalein Indikator ist, herrscht. Es dürfte auf diese Weise gelingen, eine vollkommene quantitative Analyse sämtlicher sauer reagierender Bestandteile unter Verwendung ein und desselben Indikators (= Leitfahigkeit) zu erreichen." D. Ref.) Die Azidităt nimmt proportional den zugefügten alkalisch reagierenden Salzen ab. Hopfen erhöht die Aziditat infolge der freiwerdenden Säuren und macht das Bier im Verein mit den abgegebenen Harzen haltbar.

Die Stickstoffmenge steht im engsten Zusammenhang mit der Reaktion des Maischwassers. Mäßig saure Wăsser geben eine stickstoffreichere Würze, und das um so mehr, je deutlicher der saure Charakter ist. Alkalisch reagierende Wässer reduzieren den Stickstoffgehalt, und $z$ war um so bedeutender, je höher die Alkalität ist. Neutralsalze lassen keinen Unterschied erkennen. Auch die Art der Stickstoffsubstanzen wird beeinflubt durch die Reaktion. So vermindern alkalische Wăsser nicht nur den Stickstoffgehalt, sondern sie setzen auch die Quantität der niedermolekularen Formen herab, sie erhöhen also die Quantităt der Eiweißkörper, Albumosen usw., lauter hochmolekulare Formen, sie prägen, m. a. W., die Kolloidnatur der Stickstoffsubstanzen aus und schaffen damit schlimme Zustände (D. Ref.). Saure Wässer vermehren andererseits nicht allein den Stickstoffgehalt; durch ihren Einflub wird nebenbei auch die Menge der Aminosäuren und Amine gröBer; es liegt somit in der Aziditătserhöhung ein ideales Mittel, dem Biere seinen Stickstoffsubstanzengehalt zu erhalten und zu vermehren (D. Ref.). Die Stickstoffabnahme durch Hopfenkochen (und dadurch bedingte Koagulation) war am größten bei Verwendung von Karbonatwässern.

Die Zuckermenge wird durch Karbonatwasser herabgesetzt, dagegen durch Sulfat-, Nitrat- und Chlorid-Wasser im Verhäinis der
Konzentration erhöht; neutrale Wässer scheinen keinen Effekt zur Folge zu haben.

Zum Schlusse sei noch hervorgehoben, dak alle die beschriebenen Verhältnisse vom Verfasser nur bei hellem Bier ihre volle Gültigkeit beanspruchen können, weshalb eine direkte Uebertragung auf dunkles Bier nur mit Vorsicht geschehen kann; das Allgemeine wird hier wie dort bestehen bleiben, jedoch die quantitative Seite dürfte etwas verschoben werden (D. Ref.).

P. Rohlandi2). Die Schaumbildung und Adsorption der Kolloide des Bieres.

Trotz der vielen verschiedenen Eigenschaften des Bieres, der Seife, des Eiweibes und der Talke haben diese Stoffe das eine gemeinsam: sie enthaiten Stoffe im kolloiden Zustand, sie bilden einen beständigen Schaum. Neben den zahlreichen Kolloidstoffen finden sich im Bier aus dem Hopfen stammende Säuren, Hopfen. bittersäure (Lupulinsäure), Gerbsäure usw. ; dadurch ist die Schaumbildung bedingt. Die Schaumbildung wird aufs stärkste gefördert, sobald freie Säuren oder freies Alkali oder, mit anderen Worten, Wasserstoff- oder Hydroxyllonen vorhanden sind. Sind diese Bedingungen erfüllt, so wird bei mechanischer Bewegung ein starker, andauernder und kräftiger Schaum erzeugt.

Die mechanische Bewegung wird im Falle des Bieres bei der Gärung durch die aufsteigende Kohlensäure besorgt. Bei Gelegenheit der Kohlensäure bringt der Verfasser auch uoch als vollkommen neuartig die von $H$. Freundlich und meinem Bruder (Fritz Em slander) schon längst erikannte und veröffentlichte Beobachtungstatsache, daB die Kohlensäure infolge der Adsorption durch die Bierkolloide fester gebunden ist als z. B. im Schaumwein oder in einem kohlensauren Wasser.

Fritz Emsiander ${ }^{13}$. Die Schaumhaltigkeit der Biere.

Verfasser nimmt mit Recht an, daß die EiweiBkörper zu den Trăgern der Schaumhaltigkeit zu rechnen sind und daß nicht die Quantität des Eiweißes vor allem die Schaumhaltigkeit bestimmt, sondern ausschlieflich der physikalische Zustand desselben. Dadurch ist ist es auch erklärlich, daß manchmal recht eiweiBarme Biere sehr schaumhaltend sind, während nieder vergorene und eiweibreiche Biere oft schaumios bleiben (und auch infolge

18) Zeitschr. 1. d. ges. Brauw. 1911, 320 .

19) Algen. Braver- a. Hopten-Zig. 1911, 1494. 
Kohlensäuremangel wenig rezent schmecken. D. Ref.) Nach W. Ram sden bedingt Eiweif nur dann die Schaumhaltigkeit einer Flüssigkeit, wenn es in Form von recht feinen Suspensionen zugegen ist. Diese Suspensionen sind aber auBerordentlich instabile Gebilde, d. h. sie haben beständig das Bestreben, zusammenzuflocken und dann infolge ihrer spezifischen Schwere und verzögerten Brown'schen Bewegung auszufallen. Die Hopfenharze insbesondere die Weichharze, welche beim Hopfenkochen emulgiert werden, verhindern diesen Vorgang und erhalten dadurch die neben der Azidität für die Schaumhaltigkeit äußerst wichtigen Eiweißdispersoide.

H. Bechhold. Die Kolloide in Biologie und Medizin (Dresden 1912)

Das eben erschienene Werk hat auch für den Brauer erheblichen Wert, da hier die Ergebnisse der Kolloidforschung auf die Biologie übertragen sind. Und die Biologie der Gerste gerade ist es, die dem Brauer enorm viele Schwierigkeiten bereitet, bis er dahin gelangt, aus Gerste ein gutes Malz, die Seele des Bieres, zu fertigen. Die Ausführungen $H$. Bechhold's werden dem Zymotechniker gewiB viele Klarstellungen bringen, über ein Gebiet, das bislang fast nur von Empirie erfolgreich beherrscht war.

So berichtet $z$. B. der Autor, daB durch Erschüttetungen Fermente inaktiviert werden kőnnen, ein Fingerzeig darauf, daß diesem Momente in der Brauerei, wo ja Enzyme eine hervorragende Rolle spielen, ein größeres Augenmerk geschenkt werden soll; man weiß ja schon lange, ohne aber dafür eine Erklärung gefunden zu haben, daB Erschütterungen, wie sie durch Trambahnen, Eisenbahnen u. dgl. verursacht werden, die Gärung und Lagerung nachteilig beeinflussen. Gleich wertvoll für die Brauerei ist der Hinweis, dab durch Sauerstoffmangel eine extreme Säureproduktion eintritt. "Bei der Atmung der Gerste im Keimprozesse stellt sich nach einer gewissen Zeit Sauerstoffmangel ein, besonders da die Belichtung möglichst ausgeschaltet ist; auch sorgt der Brauer selbst für Sauerstoffmangel, indem er das Malz stark, ,greifen" läBt, weil er weiB,
daB dadurch die $L \ddot{o}=u$ ug sehr gefördert wird. (Bei mangelnder Sauerstoffzufuhr tritt nun das Imbibitionswasser der Gerste als rettender Faktor auf. Das Wasser steht bekanntlich mit seinen lonen im Gleichgewicht, wenn auch nur mit einem sehr kleinen Anteil, der jedoch nach Verbrauch wieder nachgeliefert wird. Die reduzierenden Bestandteile des Malzes, die Maltose, Stärke usf. sorgen alsbald, unter Oxydation, für die Entladung der $\mathrm{OH}^{\prime}$-Ionen, während die Eiweißsubstanzen und die Diastase die $\mathrm{H}$-Ionen adsorbieren und so saure Körper werden.

Die Reaktion spielt sich folgendermaßen ab: $2 \mathrm{H}_{4} \mathrm{O}+2 \mathrm{H}+2 \mathrm{OH}^{\prime} \leftrightarrow 2 \mathrm{H}+\mathrm{O}^{\prime \prime}+\mathrm{H}_{2} \mathrm{O}$. Intramolekulare Atmung. D. Ref.)

Unter dem Kapitel ,die Nahrungs- und GenuBmittel" widmet H. B ech hold auch dem "Biere" einen breiteren Raum. Es sei daraus eine Stelle erwähnt:

"Trübe Biere sind unzulässig, hingegen kann gegen eine ,staubige' oder ,schleierige" Beschaffenheit noch kein Einwand erhoben werden. In letzterem Falle kann die disperse Phase aus Eiweibkörperchen, Dextrinen und Hopfenharzausscheidungen bestehen; auch etwas Hefe kann suspendiert sein."

Diese Ausführungen sind um so beachtenswerter, als heute das Publikum mit Unrecht an den Glanz des Bieres die allerhöchsten Anforderungen stellt und Beanstandungen, ja sogar Prozesse schon durch einen geringen „Schleier" heraufbeschworen wurden. Die Kolloidchem ic ist berufen, auch hier scharfe Grenzen zı ziehen, und $H$. Bechhold hat als ,medizinische Autorität" also das erste Wort zugunsien der Brauer gesprochen.

Schlieblich wird auch die Alkoholfrage von neuen Seiten beleuchtet. Der Autor betont auf S. 303 seines vielfach schöne Problemc enthaltenden Werkes, daB Gelatine in schwach alkoholischer Lösung stärker quillt, während umgekehrt starker Alkohol entquellend wirkt. Auf solche Weise könnte vielleicht die günstige Wirkung geringer Dosen Aikohol bei der Nahrungsaufnahme sich aufklären. 